\title{
Resolution of inflammation: state of the art, definitions and terms
}

\author{
Charles N. Serhan, ${ }^{*, 1}$ Sue D. Brain, ${ }^{\dagger}$ Christopher D. Buckley, ${ }^{\ddagger}$ Derek W. Gilroy, ${ }^{\S}$ \\ Christopher Haslett, $\|$ Luke A. J. O’Neill, ${ }^{\text {IL }}$ Mauro Perretti, ** Adriano G. Rossi, \\ and John L. Wallace ${ }^{\dagger \dagger}$ \\ *Center for Experimental Therapeutics and Reperfusion Injury, Brigham and Women's Hospital and \\ Harvard Medical School, Department of Oral Medicine, Infection and Immunity, Harvard School of \\ Dental Medicine, Boston, Massachusetts, USA; ${ }^{\dagger}$ Cardiovascular Division, King's College, New Hunt's \\ House, Guy's Campus, London, UK; ${ }^{+}$Division of Immunity and Infection, MRC Center for Immune \\ Regulation, University of Birmingham Medical School, Edgbaston, UK; ${ }^{\circledR}$ Centre for Clinical \\ Pharmacology and Therapeutics, Division of Medicine, University College London, London, UK; \\ "University of Edinburgh, MRC/Centre for Inflammation Research, The Queen's Medical Research \\ Institute, Edinburgh, UK; ${ }^{\top}$ School of Biochemistry and Immunology, Trinity College Dublin, Ireland; \\ **William Harvey Research Institute, Queen Mary University of London, Charterhouse Square, \\ London, UK; ${ }^{\dagger \dagger}$ Inflammation Research Network, University of Calgary, Calgary, Alberta, Canada
}

\begin{abstract}
A recent focus meeting on Controlling Acute Inflammation was held in London, April 27-28, 2006, organized by D.W. Gilroy and S.D. Brain for the British Pharmacology Society. We concluded at the meeting that a consensus report was needed that addresses the rapid progress in this emerging field and details how the specific study of resolution of acute inflammation provides leads for novel anti-inflammatory therapeutics, as well as defines the terms and key components of interest in the resolution process within tissues as appreciated today. The inflammatory response protects the body against infection and injury but can itself become dysregulated with deleterious consequences to the host. It is now evident that endogenous biochemical pathways activated during defense reactions can counter-regulate inflammation and promote resolution. Hence, resolution is an active rather than a passive process, as once believed, which now promises novel approaches for the treatment of inflammation-associated diseases based on endogenous agonists of resolution.-Serhan, C. N., Brain, S. D., Buckley, C. D., Gilroy, D. W., Haslett, C., O'Neill, L. A. J., Perretti, M., Rossi, A. G., Wallace, J. L. Resolution of inflammation: state of the art, definitions and terms. FASEB J. 21, 325-332 (2007)
\end{abstract}

Key Words: anti-inflammatories - leukocytes · lipid mediators - chemokines $\cdot$ cytokines

\section{KEY GELL TYPES: THE CELLULAR PLAYERS IN THE STAGE OF RESOLUTION}

In response to injury or infection, specialized, "frontline" leukocytes (polymorphonuclear neutrophils (PMNs) and eosinophils) migrate to infected/damaged sites to neutralize and eliminate potentially injurious stimuli. This requirement is perhaps the most obvious but undeniably critical one for acute inflammation to resolve. Dispensing with the inciting stimulus will halt further pro-inflammatory mediator synthesis (eicosanoids, chemokines, cytokines, cell adhesion molecules, etc.) and lead to their catabolism and the curtailment of pro-inflammatory signaling pathways (Fig. 1). Toll-like receptors are now held to play essential roles in the recognition of many of these invading organisms (vide infra). This, coupled with the release of factors that prevent ongoing PMN/eosinophil trafficking and edema formation, hails the beginning of the end-namely resolution of the acute inflammatory response and return to normal homeostasis.

One traditional view argued that pro-inflammatory mediator catabolism was sufficient for inflammation to switch off and the response subsequently just "fizzled out" (1). This is only part of the process at the tissue level, as PMN or eosinophils if left unchecked could do untold harm to an already inflamed site and must be disposed of in a controlled and effective manner. Thus, next in the sequence of events is cell clearance. The exit routes available to inflammatory leukocytes include systemic recirculation (less well described) or local death followed by their phagocytosis by recruited monocyte-derived macrophages. Once phagocytosis is complete, macrophages exit the inflamed site by lymphatic drainage with evidence that a small population may die locally by apoptosis. If all of these pathways are strictly followed, then acute inflammation will resolve

\footnotetext{
${ }^{1}$ Correspondence: Center for Experimental Therapeutics and Reperfusion Injury, Brigham and Women's Hospital and Harvard Medical School, Department of Oral Medicine, Infection and Immunity, Harvard School of Dental Medicine, 75 Francis St., Thorn 724, Boston, MA, USA 02115. E-mail: cnserhan@zeus.bwh.harvard.edu

doi: 10.1096/fj.06-7227rev
} 
Figure 1. Schematic depicting the cellular and molecular components of the inflammatory response and the requirements for resolution. A) (adapted from Cotran; see ref. 1) depicts the cellular and histological changes that occur in tissues following an inflammatory insult. Acute inflammation is characterized by the extravascular accumulation of neutrophils (PMN) and edema formation early in the response. Later during the response, mononuclear cells and macrophages accumulate and help prepare the tissue for resolution. $B, C$ ) Represent the role that specific molecular mediators play in these events. In $(B)$, we highlight that early on in the inflammatory response, immediate and early sequential predominately pro-inflammatory mediators are released, which initiate and augment the acute-phase of the response (green lights). However, this is counterbalanced by endogenous anti-inflammatory signals such as corticosterone, which serve to temper the severity and limit the duration of the early onset phase. As inflammation progresses, certain "stop signals" at appropriate "checkpoints" prevent further leukocyte traffic into tissue. These stop signals include the lipoxins, Resolvins, and prostaglandins (PGs) of the D series and pave the way for monocyte migration and their differentiation to phagocytosing macrophages, which remove dead cells and then exit the site of inflammation. Stromal cells such as fibroblasts also contribute to the resolution of inflammation by the withdrawal of survival signals and the normalization of chemokine gradients, thereby allowing infiltrating leukocytes to undergo apoptosis or leave the tissue through the draining lymphatics. This sequential set of responses leads to complete resolution and, importantly, the restoration of the inflamed tissue to its prior physiological functioning. This is the ideal sequence of events in physiological inflammation, which contrast to the situation in pathological inflammation $(B)$ where some of the factors that initiate the resolution program lead to the inappropriate accumulation of leukocytes in the wrong place at the wrong time.

without causing excessive tissue damage and give little opportunity for the development of acute, ongoing inflammation and its associated complications.

The last but equally essential aspect in the quest for tissue resolution and homeostasis is that the parenchy$\mathrm{mal} / \mathrm{stromal}$ cells that hosted the inflammatory event revert back to a non-inflammatory phenotype (2). Most current therapies target immune cells in an attempt to inhibit the production of pro-inflammatory chemical mediators. However, an equally important target is the active induction of proresolution programs by stromal cells such as fibroblasts within the inflamed tissues (2).

As with the onset phase of acute inflammation, each of the above steps in the quest for resolution is also highly coordinated and under the tight control of what may be called "proresolution" factors (3). These factors and their importance in controlling inflammation have become apparent only in the past few years (Fig. $1 A, B)$. Here, we discuss the state of the research in this field as
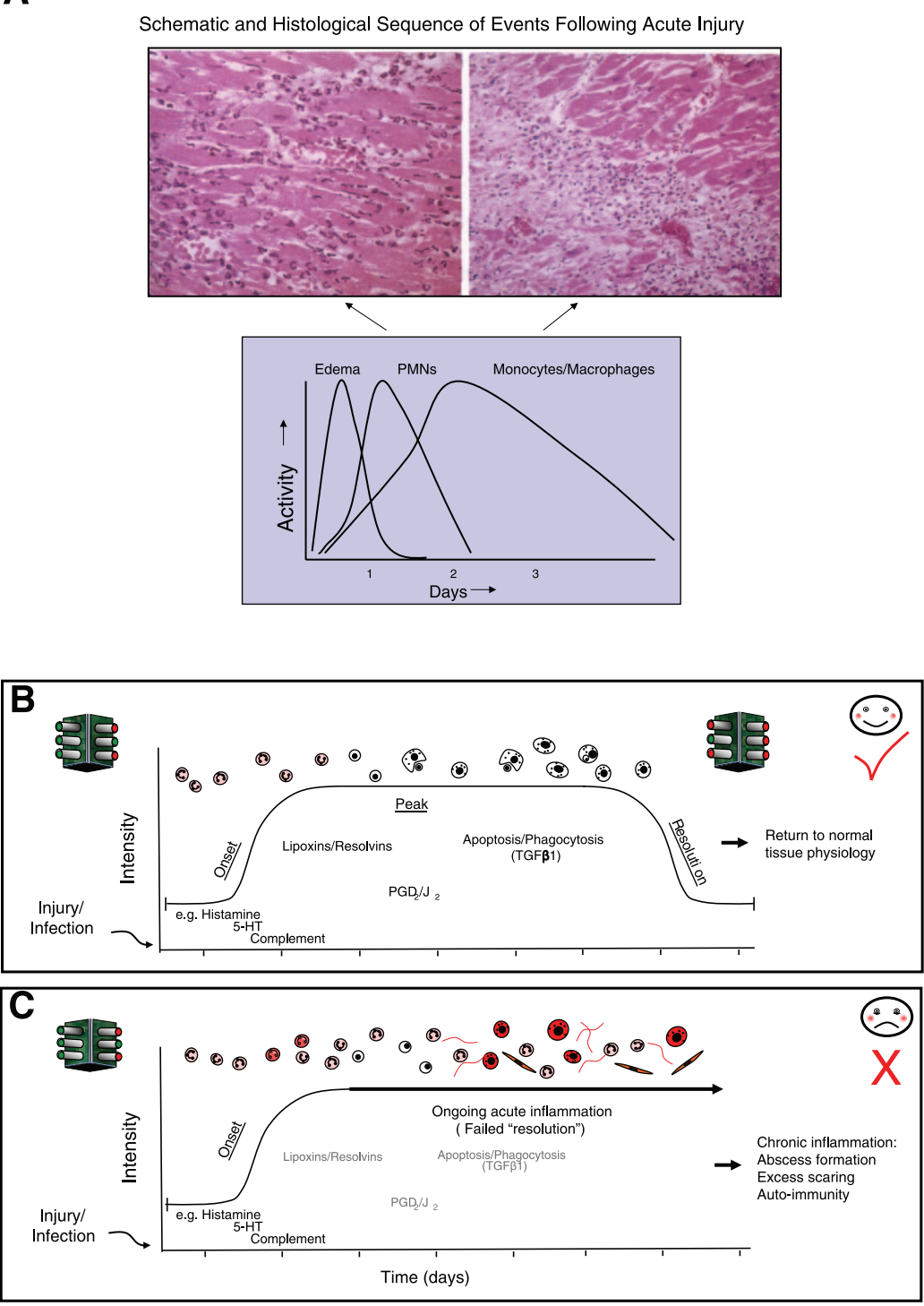

Time (days)

its stands today and highlight the virtues of "resolution" and why we trust that understanding it in molecular terms may help us in the quest for new drugs to treat inflammatory diseases.

\section{CHEMICAL MEDIATORS IN RESOLUTION}

Indeed, a number of recent reports have heightened the awareness that resolution is an active process, one that requires activation of endogenous programs that enable the host tissue to maintain homeostasis (3-5). In this paper, we shall underscore current topics and definitions of components in resolution as well as outline where gaps in information lie within this new field. It is apparent that inflammation plays an important role in the pathophysiology of many common diseases that affect Western civilizations, including some degenerative diseases not previously thought to 
have inflammatory components, such as Alzheimer's and atherosclerosis. Thus, the cellular mechanisms by which resolution occurs and the key biochemical pathways associated (8) with the return to homeostasis/ catabasis (return from disease) clearly open many new avenues for potential therapeutic interventions in a wide range of diseases associated with unresolved inflammation. These will surely aid in our understanding of innate immunity and clearance of microbes.

\section{“ANTI-INFLAMMATORY" VS. "PRO-RESOLUTION"}

The notion that the inflammatory response generates its own regulators in tandem with the better-known pro-inflammatory mediators and pathways makes sense teleologically: From the cybernetic viewpoint it is easier to control a process with both positive and negative regulatory inputs $($ Fig. $1 B)$. Or perhaps a finer level of control can be achieved; for example, consider the car metaphor of hitting the brakes to stop or the accelerator to go. Indeed, several endogenous regulators of the inflammatory response have already been elucidated (for reviews, see refs. 3-5), adding support to the idea that this is a widely employed mechanism. Clearly, disturbances in such counter-regulatory circuits could lead to exacerbated inflammatory responses just as effectively (although perhaps less obviously) than excessive activation of the pro-inflammatory cascades (6).

Noteworthy, one of the most widely used classes of anti-inflammatory and immunosuppressive drugs, the glucocorticoids, have been developed from the pioneering work of Philip Hench and represents the first successful exploitation of an endogenous anti-inflammatory mediator, cortisol.

\section{RESOLUTION OF ACUTE INFLAMMATION: WHAT WE KNOW TODAY}

Although resolution in cellular and molecular terms has been known to pathologists at the tissue level for more than 100 years, only recently have we begun to take note. Resolution of acute inflammation, or its ideal outcome, would be complete resolution. The return to homeostasis by the tissue was thought to occur by passive mechanisms. Expressly, on surgical trauma, tissue, or chemical injury, the liberated chemical mediators (exogenous and/or endogenous) would evoke leukocyte chemotaxis into tissue. The decrease in chemotactic gradients or "the burning out" of the initial signals was thought to eventually dissipate depending on the magnitude of the invading microbes and/or injury. Resolution of acute inflammation via the exodus of neutrophils from tissues after their infiltration and involvement in host defense, namely, after the job is done, was thought to be a passive series of events (Fig. $1)$. The uncovering of several distinct biochemical pathways that are actively turned on during inflammation in the resolution phase, i.e., when the numbers of neutrophils infiltrating from the tissues are dropping and are actively pushed back by the mediators produced (3), provides clear evidence for the role of active biochemical pathways in resolution.

The resolution phase can be defined at the histological level as the interval from maximum neutrophilic infiltration to the point when they are lost from the tissue. Concomitantly, mononuclear cells are then introduced in a nonphlogistic fashion and play a key role in tissue repair. They, too, are eventually lost from the tissue and are not found in tissue sections following neutralization of the insult. These cellular terms and temporal relationships $(1,7)$ have now called for the need to introduce quantitative indices, which enable us to define the precise changes in leukocytic traffic and biochemical pathways activated in exudates, as well as determine the impact of various endogenous mediators, exogenous compounds, and potential drugs within the resolution phase $(8,9)$. Along with these temporal changes in the quantity and quality of the leukocyte infiltrate, additional new approaches are needed to define how infiltrating cells change the stromal microenvironment and thereby affect the timing of tissue repair and remodeling (10).

\section{TOLL-LIKE RECEPTORS, NOD-LIKE RECEPTORS, AND RESOLUTION}

The initiation of the inflammatory response during infection or in response to sterile tissue injury involves two families of receptors, the toll-like receptors (TLRs) and nod-like receptors (NLRs) (11). TLRs recognize a range of microbial products, the best characterized is TLR4, which senses lipopolysaccharide (LPS), and TLR2, which senses bacterial lipoproteins. NLRs also sense bacterial products, the best characterized are NOD1 and NOD2, which sense peptidoglycan breakdown products, and Nalp3 (also termed cryopyrin), which is required for caspase-1 activation by several types of bacteria. Nalp3 occurs in an inflammasome complex with caspase-1, ASC, cardinal, and Nalp2 (12). The activation of the inflammasome requires TLR priming. TLRs and NLRs also sense products of inflamed tissue, and notable examples are the sensing of low MW hyaluronic acid fragments by TLR2 and TLR4 (13) and the sensing of uric acid by Nalp3 (14). Uric acid is the causative agent of gout but is also released by damaged cells, possibly as a general signal for inflammation (15). All of these represent substantial progress toward understanding innate immunity and inflammation.

Regarding resolution of inflammation, in the past two years more than 25 inhibitors of TLR and NLR action have been described, which are all induced by TLRs and therefore act as negative-feedback inhibitors (16). In effect, TLRs sow the seeds of their own destruction, and the role of negative regulators clearly indicates how robust the TLR system is. Examples include splice variants of signaling pro- 
teins such as MyD88s, cell surface receptors acting as decoys such as ST2 and SIGIRR, protein phosphatases such as MKP-1, and proteins such as Triad3a that promote degradation of TLRs (16). Deletion of genes encoding these proteins leads to a hyperinflamed state, as revealed for example by increased infiltration of neutrophils following LPS challenge in MKP-1 deficient mice (17). Similarly, inhibitors of NLRs have been found, such as caspase recruitment domain (CARD)-only protein (COP) or inhibitor of pro-caspase-1 activation (ICEBERG).

Once TLRs or NLRs are triggered, their effects will ultimately be limited, allowing resolution to proceed. It is also possible that inflammation will become chronic, however, if the system is somehow dysregulated. An example here is the work of Noble and colleagues, who have shown that polymeric hyaluronic acid binds TLR2 and TLR4 and generates a protective signal in the epithelium in lung (13). Fragments of hyaluronic acid, however, generated in response to tissue injury, become inflammatory, again via TLR2 and TLR4 via an unknown mechanism. This in turn will lead to the further breakdown of hyaluronic acid via induction of hyaluronidases, promoting further inflammation. This might turn into a vicious circle, leading to chronicity, particularly if there is a defect in negative regulation due to polymorphisms in the negative regulator.

Another example concerns regulatory $\mathrm{T}$ cells ( $\mathrm{T}$ regs) $(18,19)$. These cells inhibit immunity and also suppress inflammation. During bacterial infection, TLR2 ligands are sensed by TLR2 on both T regs and macrophages. The $\mathrm{T}$ regs expand but are kept inhibited. TLR2 in macrophages promotes host defense, which leads to bacterial clearance. Once the TLR2 ligands have been removed, the "brake" is removed from the $T$ regs. They produce IL10, limit inflammation, presumably promote resolution, and importantly prevent autoimmunity. These recent results provide insight into the resolution process and how it might become dysregulated, leading to chronic inflammation.

\section{HOW TARGETING RESOLUTION RATHER THAN INHIBITING INFLAMMATION WOULD AFFECT DRUG DISCOVERY PROGRAMS}

Current anti-inflammatory therapy arises from our need to control the cardinal signs of inflammation. Most of the anti-inflammatories studied to date block/ antagonize key pro-inflammatory mediator pathways that are elicited on the initiation of an acute inflammatory response. Thus, most of the anti-inflammatories in use block key biochemical pathways and/or block the signaling of key pro-inflammatory mechanisms.

With the past few years has been a growing recognition that, although targeting infiltrating immune cells can control the inflammatory response, it does not lead to remission or permanent resolution. Inflammatory reactions occur within distinct micro-environments composed of tissue-specific cells, such as fibroblasts, endothelial cells, and macrophages, along with their highly specialized extracellular matrix (ECM) components. Evidence now exists that such stromal cells can determine the nature and duration of leukocyte infiltrates (20). At the conclusion of the inflammatory response, stromal cells also contribute to resolution of inflammation by $i$ ) the withdrawal of survival signals, ii) the normalization of chemokine gradients, and iii) the induction of resolution programs that allow infiltrating cells to undergo apoptosis or exit the inflamed tissue through draining lymphatics. The subversion of these pathways would lead to persistent inflammation, which can be remarkably stable. The relative lack of reagents that target the stromal cells may account for the failure of current treatments to affect a permanent cure.

Embracing resolution would require a cultural shift in emphasis from depletion therapy to replacement therapy (i.e., as in endocrinology where a missing agent, e.g., insulin, is replaced). Currently, anti-inflammatory approaches target chemical mediators generated during the resolution phase (as defined above, temporally from surrounding tissues or by the traffic of specific leukocytes, neutrophils, monocytes, and macrophages in and out of the tissue). Resolution therapies act quite differently in that they serve as activators, e.g., receptor agonists, which turn on protective mechanisms that lead to the return of homeostasis.

Consistently, agonists at receptor targets utilized by endogenous anti-inflammatory proresolving mediators exert an anti-inflammatory effect when added back into experimental models/specific settings in the laboratory $(4,5)$. We envision that new drugs specifically designed to promote resolving mediators and biochemical pathways will have a much better compliance and be "homeostatic and modulatory" in their actions, mimicking the way inflammation naturally subsides in the body. Hence, it is highly likely that these new proresolving drugs would be better tolerated.

\section{"RESOLUTION TOXIC" AND "RESOLUTION FRIENDLY" DRUGS ALREADY IN OUR MIDST}

It is now very apparent that certain widely used drugs, as well as those in experimental settings, are "resolution-toxic" in that they derange or impair timely and/or complete resolution. Since no widely established in vivo models monitored the key components in resolution, a set of resolution indices were recently introduced to help set benchmarks for assessing the impact of proresolving agents and their impact within resolution (8). Many currently used drugs and potential therapeutic compounds without undesirable effects have slipped by underappreciated.

This is most notable in the case of the inhibition of COX-2 with selective COX-2 inhibitors, where these inhibitors block the production of $\mathrm{PGE}_{2}$ and $\mathrm{PGD}_{2}$, which play important roles in bringing about resolution (21). Their findings also underscore the role of COX-2 and its products in both the initiation of acute inflam- 
matory response as well as its resolution. Thus, temporal and spatial positioning of these biochemical pathways can dictate the actions of their local mediators. Hence, it is important to develop drugs that spare the resolution process. Besides COX-2 inhibitors, another example may be anti-TNF therapy. It has been proposed that the poor clinical outcome observed in SIRS patients is related to the derailment of a proper temporal anti-inflammatory proresolving response.

An ideal drug to treat inflammatory disease would be able to both blunt the inflammatory response as well as activate the resolution program. Glucocorticoids, in addition to their anti-inflammatory properties, also promote resolution by stimulating macrophages in a nonphlogistic proresolving fashion to stimulate the uptake of apoptotic neutrophils (22), possibly by altering the phenotype of macrophages during their differentiation via a mechanism involving reorganization of key cytoskeletal elements (23) and/or acting via another endogenous homeostatic anti-inflammatory factor, annexin 1 (24).

Additionally, the widely used aspirin jump-starts resolution by making up for its loss in prostaglandin production, which plays key roles in resolution, by instead generating the epimeric version of lipid mediators (25), such as the aspirin-triggered lipoxins (recently reviewed in ref. 26), as well as specific epimeric versions of the omega-3 fatty acid-derived resolvins and protectins. Perhaps these drugs are so widely used today because of a perceived reduction in the cardinal signs of inflammation and ergo their anti-inflammatory actions. However, in addition they spare and/or enhance resolution. Thus, it is important for us to clearly define the earlier signs of resolution in molecular terms, which at present do not appear to be effectively captured in clinical endpoints. This will be key in appreciating and selecting the most appropriate anti-inflammatory therapies to use in a given clinical scenario.

Recent evidence has shown aspirin-triggered lipoxins and glucocorticoid-induced annexin 1 converging onto an identical receptor target, a specific 7-transmembrane spanning G-protein coupled receptor (27). Thus, as in the case of pro-inflammatory mediators and the long list of cascades interlinking them (think of cytokines and chemokines), very recent work highlights the existence of an anti-inflammatory network, whereby distinct proresolving mediators and pathways are interconnected to ensure the correct resolution of inflammation and return of homeostasis. Besides lipoxins and annexin 1, another example is that of melanocortins or nuclear receptor agonists and heme oxygenase-1. These endogenous mediators qualify as agonists of key steps in resolution. Hence, they are proresolving mediators with anti-inflammatory properties in vivo. It is likely that other drugs in the general practitioner's armamentarium would act, at least in part, by promoting or mimicking proresolution pathways, but this point might have been totally overlooked because it has not been investigated at all!

Currently no drugs in the clinic are purposefully based on the elicitation of proresolving pathways, although compounds that target apoptotic pathway may have potential. Recently, it was shown in a rat carrageenan-induced pleurisy model of resolving inflammation that manipulation of key signaling pathways influenced resolution (28). In that study, an inhibitor (PD98059) of MAPK (ERK1/2), administered at the peak of inflammation, promoted resolution by enhancing inflammatory cell apoptosis. Conversely, administration of a specific inhibitor (V5) of the proapoptotic Bcl-2 family member, Bax, attenuated resolution by inhibiting inflammatory cell apoptosis. In another study (29), it was demonstrated that inhibitors of phosphoinositide-3 kinase, namely wortmannin and LY294002, administered at the peak of a resolving model of allergic pleurisy in mice markedly enhanced the clearance of eosinophils by promoting their apoptosis and their subsequent removal. However, we would like to stress that if apoptosis is induced specifically in inflammatory cells, it must be accompanied with efficient macrophage clearance of the apoptotic cells. If this did not occur, it is likely that failed clearance of the apoptotic cells would result in secondary necrosis and result in unwanted tissue damage. It may be that macrophages, or other cells with phagocytic potential (e.g., fibroblasts, epithelial cells, etc.), have the capacity to deal with induced apoptosis. Fortunately, macrophage phagocytosis is regarded as a highly efficient and regulatable process and can therefore be modulated by pharmacological means as occurs with glucocorticoids and lipoxins $(3,24)$.

There are, however, drugs that mediate their effects, at least in part, by triggering the synthesis of endogenous anti-inflammatory mediators, counteracting the activity of early pro-inflammatory "go signals" to prepare for or facilitate resolution. These include methotrexate, sulfasalazine, and FK506, which are thought to exert their effects by increasing adenosine synthesis, another endogenous mediator released very early in the inflammatory response and acting through the $\mathrm{A} 2 \mathrm{~A}$ receptor to control leukocyte trafficking by elevating intracellular levels of cAMP (30). However, glucocorticoids, while acting on many facets of the inflammatory response, traditionally by curtailing the severity of onset, may also exert hitherto unknown bona fide proresolution effects through their ability to hasten apoptosis in certain leukocytes (e.g., eosinophils and lymphocytes) while concomitantly enhancing the ability of monocyte-derived macrophages to phagocytose apoptotic cells. One may argue that, given that steroids are used mainly after the inflammatory event has started, their therapeutic benefits arise largely from eliciting proresolution pathways.

\section{CLINICAL IMPLICATIONS OF RESOLUTION}

What are the clinical implications of nonresolving inflammation? Traditionally, clinicians have considered certain forms of inflammation nonresolving, as in the 
case of chronic Pseudomonas infections experienced in cystic fibrosis, a classic nonresolving inflammation/ infection (1). However, in many clinical conditions infection does not seem to play a role in the persistence of the disease (i.e., chronic persistent infection is not the same as chronic persistent inflammation) (7). These include most if not all of the immune-mediated inflammatory diseases: rheumatoid arthritis, asthma, inflammatory bowel disease, and psoriasis. In these conditions, the persistence of the inflammatory response appears to have become divorced from the inciting agent. Classic anti-inflammatory agents only provide symptomatic relief and do not alter the course of the disease.

It is highly likely that this is because the naturally occurring resolution programs have been subverted in such diseases. The problem is that current clinical measurements do not adequately capture or even measure how inflammation differs from repair and resolution (8). Most clinical parameters take account of disease activity and accumulated damage, but very few can measure resolution and repair. This finding suggests that we have been using the wrong measurements and that new clinical and pathological indices will need to be developed to measure these parameters effectively. It is possible that a number of useful drugs have been discarded because they did not show efficacy using classic anti-inflammatory readouts; yet they may have been very effective in promoting resolution. Thus, the temporal phase of the disease will determine whether a drug is likely to work. For example, consider that TGF- $\beta$ is anti-inflammatory during the initial phase of an inflammatory response yet at the later phases it prolongs inflammation, so that replacement might be more important than blockade depending on the phase of the disease.

\section{KINETICS AND THE INVESTIGATOR}

An experimental setting at time zero with addition of a pro-inflammatory agonist and/or a challenge permits us as experimentalists to document precise kinetic events and their temporal relationships in animal disease models in vivo. It is difficult to capture these models with isolated cell systems in order to get a handle on the components of resolution or, for example, determine whether compounds are proresolving $(8,20-24)$. Moreover, it is difficult to spot these in clinical situations because patients with active disease do not arrive in the clinic at time zero. Therefore, it is important to recognize early molecular components to study them in these individuals for cause-and-effect relationships.

It is becoming increasingly clear that inflammation has a number of tactically placed checkpoints that limit the magnitude and duration of the response. Defects in these endogenous anti-inflammatory pathways will arguably predispose the host to chronic inflammatory diseases (Fig. 1C). Of these endogenous controllers, the cyclooxygenase and lipoxygenase (LOX) pathways have both protective and pro-inflammatory roles in inflammation, with the precise function each enzyme plays depending on the inflammatory stimulus, the phase of the acute inflammatory response when these enzymes are expressed (31), and whether it is resolving or nonresolving inflammation. This raises a number of important matters pertaining to anti-inflammatory drug development. We need to be mindful that during inflammation endogenous pathways may attempt to limit or switch off the ongoing response. These pathways must not be inadvertently altered by anti-inflammatory drugs (Fig. 1B,C). The flip-side of this biological scenario is the realization that factors expressed during resolution could be mimicked and used to push ongoing inflammation down a proresolution pathway $(8,31)$. Such an approach would mean developing molecules that exert multiple effects at various phases of the inflammatory response-limiting leukocyte trafficking, hastening cell clearance, and helping to restore inflamed stromal tissue to its prior state. Though a chemist's nightmare, it could arguably exert striking effects on ongoing inflammatory diseases. Although we don't develop drugs along these lines, it is provocative to think that existing anti-inflammatory agents such as steroids, whose development was based on an entirely different strategy, may exert potent proresolution effects arising from their proapoptotic (eosinophils) and prophagocytic properties.

The potential side effect of developing "proresolvers" (and it seems that not all treatments are without side effects) is that inflammation could be prematurely resolved before it has a chance to deal with the insulting agent, the very essence of the inflammatory response (26) in the first instance. This brings us to underlining the fact that all inflammatory responses are not the same and that developing "pan-inflammatory inhibitors" is not necessarily the most effective approach to treating inflammation-driven diseases. Thus, we need to foster a greater understanding of the nature of the response that drives each individual inflammatory event and its resolution and tailor treatments accordingly. To achieve this goal we must improve our understanding of the mechanisms in resolution of inflammation and identify possible approaches to promote this process, perhaps in combination with antiinflammatory therapy. Of interest, pharmacological induction of caspase-dependent inflammatory cell apoptosis by selective cyclin-dependent kinase (CDK) inhibitors can augment the resolution of established inflammation (32). It was shown that human neutrophils contain functionally active CDKs (CDK1, 2, and 5) and that different CDK inhibitor drugs induce neutrophil apoptosis per se and override the retardation of apoptosis induced by survival factor such as GM-CSF. In addition, the specific CDK inhibitor, R-roscovitine (the anti-cancer agent), enhanced inflammatory resolution in three in vivo mouse models of established neutrophil-dependent inflammation (carrageenan-elicited acute pleurisy, bleomycin-induced lung injury, and 
passively-induced arthritis). It was also demonstrated that the CDK inhibitor drug-induced enhanced resolution of established pleurisy was mediated by caspasedependent apoptosis of inflammatory cells. Thus, it is possible that CDK inhibitor drugs may provide a novel strategy for enhancing the resolution of inflammation and may have potential for the treatment of inflammatory disorders.

At present, it appears that possible deficiencies in resolution pathways and/or precursors of proresolving mediators, such as dietary essential omega-3 fatty acids, which are precursors to resolvins and protectins (Fig. $1 C)$, may lead to prolonged inflammation and the inability for the tissue to return to homeostasis (26) and complete the full life-cycle per se of the neutrophils and mononuclear cells recruited to the tissue.

Therefore, it is very likely that the diffused application of unbiased wide-spectrum approaches (i.e., microarrays, lipid mediator informatics, proteomics) in experimental (8) and clinical trials will unveil important and "unexpected" links between novel therapeutics still under development-and likely old drugs, too-and induction of endogenous anti-inflammatory mediators. We are at the dawn of a new era of drug discovery for inflammatory diseases, which finally exploit natural endogenous mechanisms operating in our body to terminate the inflammatory response. The present consensus will hopefully provide a framework to facilitate our appreciation of these resolution components and their structure-function that are key to this process for navigating resolution to improve health. FJ

The authors acknowledge grant support for their respective research efforts from the National Institutes of Health (U.S.A.) and Medical Research Councils (U.K., Ireland, Canada). We also thank Mary Haim Small and Stacey Lindberg (CET\&RI, Brigham and Women's Hospital) for their expert assistance with the preparation of this manuscript.

\section{REFERENCES}

1. Cotran, R. S., Kumar, V., and Collins, T., eds. (1999) Robbins Pathologic Basis of Disease, W.B. Saunders Co., Philadelphia, Pennsylvania, USA

2. Filer, A., Pitzalis, C., and Buckley, C. D. (2006) Targeting the stromal microenvironment in chronic inflammation. Curr. Opin. Pharmacol. 6, 393-400

3. Serhan, C. N. (2004) A search for endogenous mechanisms of anti-inflammation uncovers novel chemical mediators: missing links to resolution. Histochem. Cell Biol. 122, 305-321

4. Gilroy, D. W., Lawrence, T., Perretti, M., and Rossi, A. G. (2004) Inflammatory resolution: new opportunities for drug discovery. Nat. Rev. Drug. Discov. 3, 401-416

5. Perretti, M. (1997) Endogenous mediators that inhibit the leukocyte-endothelium interaction. Trends Pharmacol. Sci. 18, $418-425$

6. Weissmann, G., Smolen, J. E., and Korchak, H. M. (1980) Release of inflammatory mediators from stimulated neutrophils. N. Engl. J. Med. 303, 27-34

7. Majno, G., and Joris, I. (2004) Cells, Tissues, and Disease: Principles of General Pathology, Oxford Univ. Press, New York, New York, USA

8. Bannenberg, G. L., Chiang, N., Ariel, A., Arita, M., Tjonahen, E., Gotlinger, K. H., Hong, S., and Serhan, C. N. (2005) Molecular circuits of resolution: Formation and actions of resolvins and protectins. J. Immunol. 174, 4345-4355

9. Damazo, A. S., Yona, S., Flower, R. J., Perretti, M., and Oliani, S. M. (2006) Spatial and temporal profiles for anti-inflammatory gene expression in leukocytes during a resolving model of peritonitis. J. Immunol. 176, 4410-4418

10. Parsonage, G., Filer, A. D., Haworth, O., Nash, G. B., Rainger, G. E., Salmon, M., and Buckley, C. D. (2005) A stromal address code defined by fibroblasts. Trends. Immunol. 26, 150-156

11. Creagh, E., and O'Neill, L. A. (2006) TLRs, NLRs and RLRs: a trinity of pathogen sensors that co-operate in innate immunity. Trends Immunol. 352-357

12. Agostini, L., Martinon, F., Burns, K., McDermott, M. F., Hawkins, P. N., and Tschopp, J. (2004) NALP3 forms an IL-1beta-processing inflammasome with increased activity in Muckle-Wells autoinflammatory disorder. Immunity 20, 319-325

13. Jiang, D., Liang, J., Fan, J., Yu, S., Chen, S., Luo, Y., Prestwich, G. D., Mascarenhas, M. M., Garg, H. G., Quinn, D. A., et al. (2005) Regulation of lung injury and repair by toll-like receptors and hyaluronan. Nature Med. 11, 1173-1179

14. Martinon, F., Petrilli, V., Mayor, A., Tardivel, A., and Tschopp, J. (2006) Gout-associated uric acid crystals activate the NALP3 inflammasome. Nature 440, 237-241

15. Shi, Y., Evans, J. E., and Rock, K. L. (2003) Molecular identification of a danger signal that alerts the immune system to dying cells. Nature 425, 516-521

16. Liew, F. Y., Xu, D., Brint, E. K., and O'Neill, L. A. (2005) Negative regulation of toll-like receptor-mediated immune responses. Nat. Rev. Immunol. 5, 446-458

17. Sonya, M. A., et al. (2006) Anti-inflammatory effects of dexamethasone are partly dependent on induction of dual specificity phosphatise-1 (DUSP1). J. Exp. Med., in press

18. Liu, H., Komai-Koma, M., Xu, D., and Liew, F. Y. (2006) Toll-like receptor 2 signalling modulates the functions of CD4+ CD25+ regulatory T cells. Proc. Natl. Acad. Sci. U. S. A. 103, 7048-7053

19. Sutmuller, R. P., den Brok, M. H., Kramer, M., Bennink, E. J., Toonen, L. W., Kullberg, B. J., Joosten, L. A., Akira, S., Netea, M. G., and Adema, G. J. (2006) Toll-like receptor-2 controls expansion and function of regulatory T cells. J. Clin. Invest. 116, 485-494

20. Buckley, C. D., Pilling, D., Lord, J. M., Akbar, A. N., ScheelToellner, D., and Salmon, M. (2001) Fibroblasts regulate the switch from acute resolving to chronic persistent inflammation. Trends. Immunol. 22, 199-204

21. Gilroy, D. W., Colville-Nash, P. R., Willis, D., Chivers, J., Paul-Clark, M. J., and Willoughby, D. A. (1999) Inducible cycloxygenase may have anti-inflammatory properties. Nature Med. 5, 698-701

22. Liu, Y., Cousin, J. M., Hughes, J., Van Damme, J., Seckl, J. R., Haslett, C., Dransfield, I., Savill, J., and Rossi, A. G. (1999) Glucocorticoids promote nonphlogistic phagocytosis of apoptotic leukocytes. J. Immunol. 162, 3639-3646

23. Giles, K. M., Ross, K., Rossi, A. G., Hotchin, N. A., Haslett, C., and Dransfield, I. (2001) Glucocorticoid augmentation of macrophage capacity for phagocytosis of apoptotic cells is associated with reduced p130Cas expression, loss of paxillin/pyk2 phosphorylation, and high levels of active Rac. J. Immunol. 167, 976-986

24. Maderna, P., Yona, S., Perretti, M., and Godson, C. (2005) Modulation of phagocytosis of apoptotic neutrophils by supernatant from dexamethasone-treated macrophages and annexinderived peptide Ac(2-26). J. Immunol. 174, 3727-3733

25. Serhan, C. N., Hong, S., Gronert, K., Colgan, S. P., Devchand, P. R., Mirick, G., and Moussignac, R.-L. (2002) Resolvins: a family of bioactive products of omega-3 fatty acid transformation circuits initiated by aspirin treatment that counter proinflammation signals. J. Exp. Med. 196, 1025-1037

26. Serhan, C. N., and Savill, J. (2005) Resolution of inflammation: the beginning programs the end. Nature Immunol. 6, 1191-1197

27. Perretti, M., Chiang, N., La, M., Fierro, I. M., Marullo, S., Getting, S. J., Solito, E., and Serhan, C. N. (2002) Endogenous lipid- and peptide-derived anti-inflammatory pathways generated with glucocorticoid and aspirin treatment activate the lipoxin A(4) receptor. Nat. Med. 8, 1296-1302

28. Sawatzky, D. A., Willoughby, D. A., Colville-Nash, P. R., and Rossi, A. G. (2006) The involvement of the apoptosis-modulating proteins ERK 1/2, Bcl-xL and Bax in the resolution of acute inflammation in vivo. Am. J. Pathol. 168, 33-41 
29. Pinho, V., Souza, D. G., Barsante, M. M., Hamer, F. P., De Freitas, M. S., Rossi, A. G., and Teixeira, M. M. (2005) Phosphoinositide-3 kinases critically regulate the recruitment and survival of eosinophils in vivo: importance for the resolution of allergic inflammation. J. Leukoc. Biol. 77, 800-810

30. Cronstein, B. N. (2006) Going with the flow: methotrexate, adenosine, and blood flow. Ann. Rheum. Dis. 65, 421-422

31. Levy, B. D., Clish, C. B., Schmidt, B., Gronert, K., and Serhan, C. N. (2001) Lipid mediator class switching during acute inflammation: signals in resolution. Nature. Immunol. 2, 612-619
32. Rossi, A. G., Sawatzky, D. A., Walker, A., Ward, C., Sheldrake, T. A., Riley, N. A., Caldicott, A., Martinez-Losa, M., Walker, T. R., Duffin, R., et al. (2006) Cyclin-dependent kinase inhibitors enhance the resolution of inflammation by promoting inflammatory cell apoptosis. Nat. Med. 12, 1056-1064

Received for publication August 28, 2006. Accepted for publication September 22, 2006. 